\title{
LES GARANTIES JURIDIQUES DE L'ALTERNANCE POLITIQUE DANS LES CHARTES CONSTITUTIVES DES ORGANISATIONS INTERNATIONALES AFRICAINES. CAS DE L'UA, DE LA SADC ET DE LA CIRGL
}

\author{
Par Joseph CIHUNDA HENGELELA ${ }^{1}$
}

\section{Résumé}

Cet article retrace le parcours du principe de l'alternance politique dans les Etats africains comme un principe à vocation d'une norme de droit international. Consacré dans des déclarations des chefs d'Etat et de gouvernement, le principe de l'alternance est en train d'être intégré dans les instruments juridiques contraignants des organisations internationales africaines. Cet article se projette sur l'avenir ce principe et de sa contribution à l'édification d'un Etat de droit réellement démocratique dans les Etats africains.

\begin{abstract}
This article traces the career of the principle of political alternance in African states as a principle aim of a norm of international law. Enshrined in the declarations of the Heads of State and Government, the principle of alternance is being integrated into legally binding instruments of African international organizations. This article projecting the future this principle and its contribution to the building of a truly democratic state of law in African states.
\end{abstract}

\section{Introduction}

2016 une année électorale en Afrique avec six scrutins présidentiels ${ }^{2}$. Elle constitue la charnière entre les années 2015 et 2017 concernées par des élections dans certains Etats où la consolidation de l'Etat de droit donnera une lueur d'espoir pour la paix. En rapport avec la

1 Diplômé d'Etudes Supérieures en Droit Public et Doctorant/Université de Kinshasa et membre du Conseil pour le développement de la recherche en sciences sociales en Afrique (CODESRIA), du Centre des Recherches et d'Etudes sur l'Etat de Droit en Afrique (CREEDA) et de Legal Research Team of African Mining Legislation Atlas (LRT/AMLA/WBG). Consultant et Avocat au Barreau de Kinshasa/Matete, Email : josephcihunda@gmail.com; Tél. : 00243810399060.

2 Chougar et Charpentier, «Les grands rendez-vous électoraux en Afrique en 2016 », http://www.rfi. fr/afrique/20160106-grands-rendez-vous (Consulté, le 10 mars 2016). Ces Etats sont : RCA, Ouganda, Niger, Bénin, Comores, Congo-Brazzaville, Tchad, Djibouti, Sao Tomé et Principe, Gabon, CapVert, Zambie, Guinée Equatoriale, Gambie, RDC et Ghana. 
problématique de l'alternance politique, les élections présidentielles de 2016 dans certains Etats africains comme le Bénin, la RDC, le Congo-Brazzaville, le Gabon, le Tchad devaient permettre de tourner la page de certains règnes présidentiels ${ }^{3}$. En effet, au Bénin, en RDC et au Congo Brazzaville, l'alternance politique est assurée par le mécanisme de limitation constitutionnelle des mandats présidentiels. Au Bénin, le Président sortant a respecté la Constitution. En RDC, le Président Joseph Kabila Kabange, après plusieurs tentatives de révision ou de changement de constitution, garde un mutisme qui cache très mal sa volonté de se maintenir au pouvoir au lendemain de son deuxième et ultime mandat ${ }^{4}$. Au CongoBrazzaville, le Président Denis Sassou Ngouesso a réussi le « coup de maître » en changeant de constitution pour se maintenir au pouvoir après 32 ans de règne.

Au Gabon et au Tchad, les Présidents de la République en fonction n'ont pas beaucoup d'inquiétudes à se faire. Ils ne sont pas soumis à la contrainte constitutionnelle de limitation des mandats présidentiels. Il en est aussi le cas pour l'Ouganda où après 30 ans au pouvoir le Président Yorewi Kaguta Museveni a été réélu à l'occasion d'un processus électoral inéquitable et non transparent ${ }^{5}$. Les Présidents de la République du Togo, du Cameroun, de la Gambie $^{6}$, de la Guinée Equatoriale, de l'Angola et autres Etats tirent largement le profit de ce qui paraît comme une tare dans la gouvernance des Etats en Afrique.

Le tableau que l'on peut dresser sur l'alternance politique en Afrique n'est pas que sombre. Des lueurs d'espoirs et des signes encourageants sont à mentionner. Le Sénégal qui avait donné le premier exemple d'alternance politique en 1980 poursuit bon gré mal gré sa trajectoire. La Tanzanie n'a pas cessé d'étonner en se constituant en une étoile démocratique en Afrique de l'Est. Il en est de même de nombreux Etats en Afrique australe.

L'alternance politique est finalement devenue une composante essentielle du maintien de la paix au sein des Etats et au niveau régional. Elle est un élément fondamental de l'Etat de droit et une preuve de la vitalité d'une démocratie. L'objectif de la présente étude est de rechercher dans les chartes constitutives des organisations internationales africaines les bases juridiques de l'alternance politique au sein des Etats. La mise en exergue ces fondements présente l'avantage de servir d'argumentaire solide pour l'action internationale en faveur de l'alternance politique en Afrique.

$3 \mathrm{Au}$ Gabon à la suite d'une élection jugée par les observateurs indépendants non transparente, au Tchad sur fond de la violence et de la contestation.

4 Un dialogue politique est en cours et à pour finalité de prolonger le mandat du Président de la République à cause du fait que les élections ne peuvent pas être organisées dans le délai constitutionnel. Le projet de cet accord politique s'il venait d'être adopté tel quel il empêchera la première alternance démocratique et pacifique dans ce pays.

5 Peter Girke \& Mathias Kamp, Museveni's Uganda eternal subscription for power?, in KAS International reports, Volume 27, 2011, pp. 49-70. Angelika Klein, Uganda's Youth. Opportunities and Challenges in the second youngest country in the word ", in KAS International reports, Volume 29, 2013, pp. 26-40.

6 Francis Kpatindé, « Yahya Jammeh: un autocrate fantasque à la tête de la Gambie », http://www.rfi. fr/afrique/20150105-yahya-jammeh-autocrate (Consulté 10 mars 2016). 
L'analyse de ce sujet se situe dans une approche pyramidale partant des instruments juridiques de l'UA pour se terminer au niveau des Communautés économiques régionales auxquelles appartient la République Démocratique du Congo.

\section{A. L'Union africaine (UA) et l'alternance politique dans les Etats membres}

Par rapport à l'OUA, l'UA a marqué une différence notable au regard de la gouvernance démocratique dans les Etats membres. Sur le plan de principes, l'UA promeut l'alternance politique à travers son Acte constitutif et dans la Charte africaine de la démocratie, des élections et de la gouvernance (CADEG). Tous ces instruments ont été adoptés dans le contexte de l'émergence démocratique et de l'Etat de droit en Afrique.

\section{L'alternance politique de l'Acte constitutif de l'Union africaine (UA)}

Dans l'Acte constitutif de l'UA, les Etats membres se sont engagés à promouvoir les principes et les institutions démocratiques ${ }^{7}$. Ils se sont également engagés à œuvrer notamment pour le respect des principes démocratiques et de l'Etat de droit ${ }^{8}$. L'une des conséquences de cet engagement est la condamnation et le rejet des changements anticonstitutionnels de gouvernement ${ }^{9}$. Ces engagements ont été proclamés d'abord à Lomé puis réitérés lors du premier sommet de la Conférence de l'UA à Durban en Afrique du Sud en 2002.

La particularité de ce sommet est que la Déclaration sur la gouvernance démocratique qui y a été faite peut servir d'instrument d'interprétation des engagements de membres de l'UA en faveur de l'alternance politique. La Déclaration de Durban réaffirme la volonté des dirigeants africains de promouvoir «le droit inaliénable de l'individu à participer, par le biais de processus politiques et démocratiques libres et crédibles, à l'élection périodique de ses dirigeants pour des mandats déterminés ${ }^{10} \ldots »$.

Les termes « élection périodique » et " mandats déterminés 》 repris dans cette déclaration font allusion assurément à l'alternance politique démocratique. Cette opinion est secourue par la même déclaration lorsque les chefs d'Etat et de gouvernement s'engagent à

7 Art. 3 point 7 de l'Acte constitutif de l'UA, Lomé, le 11 juillet 2000.

8 Art. 4 point 13 de l'Acte constitutif de l'UA...op. cit.

9 Art. 4 point 16 de l'Acte constitutif de l'UA...op. cit. Voir dans ce sens, Sayeman Bula-Bula, Mise hors-la-loi ou mise en quarantaine des gouvernements anticonstitutionnels par l'Union africaine?, African Yearbook of International Law, 2005, pp. 23-78; Joseph Kazadi Mpiana, L’Union africaine face à la gestion des changements anticonstitutionnels de gouvernement, Revue québecoise de droit international, $\mathrm{n}^{\circ}$ 25, février 2012; Paulin Punga Kumakinga, L'interdiction des changements anticonstitutionnels de gouvernement en Afrique: pratique, bilan et perspectives, in Oswald Ndeshyo Rurihose (ed.), Le nouvel élan du panafricanisme, l'émergence de l'Afrique et la nécessité de l'intégration continale: Les actes des journées scientifiques consacrées à la commémoration de la journée de l'Afrique: 2011-2012-2013-2014, Kinshasa, 2014, pp. 425-457.

$10 \S 7$ de la Déclaration de l'UA sur la gouvernance démocratique, politique, économique et des entreprises, Durban, 2002. 
veiller à ce que les « constitutions nationales respectives reflètent les idéaux démocratiques » et acceptent d'appliquer « rigoureusement la position de l'Union africaine (UA) sur les changements anticonstitutionnels de gouvernement ${ }^{11} »$.

Toujours à Durban et au cours de la même période, les Etats membres de l'UA avaient adoptés le Mémorandum d'accord sur la sécurité, la stabilité, le développement et la coopération en Afrique. Dans cet instrument de politique générale, il fait allusion à la limitation des mandats des responsables politiques. En effet, les Etats africains s'engageaient à

Adopter, d'ici 2005, un Code de conduite à l'endroit des responsables politiques prévoyant, entre autres, une limitation constitutionnelle du mandat des responsables politiques élus, basé sur le renouvellement de leur mandat; les gouvernements devront se conformer scrupuleusement à ces règles ${ }^{12}$.

Bien avant, les chefs d'Etats africains avaient nommément, dans la Déclaration de Lomé du 12 juillet 2000, inséré l'alternance démocratique parmi les principes de la gouvernance en Afrique $^{13}$. Ces deux Déclarations ont servi de fondement politique à l'élaboration de la Charte africaine de la démocratie, des élections et de la gouvernance.

\section{L'alternance politique dans la Charte africaine de la démocratie, des élections et de la} bonne gouvernance (CADEG)

Cette volonté de promouvoir l'alternance politique apparaît clairement dans la CADEG. Dès le préambule de cette Charte, le souci d'enraciner une culture d'alternance politique fondée sur la tenue régulière d'élections transparentes, libres et justes est exprimé par les dirigeants africains ${ }^{14}$. C'est pourquoi la promotion et le renforcement de l'adhésion au principe de l'Etat de droit fondé sur le respect et la suprématie de la Constitution et de l'ordre constitutionnel dans l'organisation politique des Etats africains est un des objectifs poursuivis par la Charte $^{15}$.

Entrée en vigueur le 15 février 2012, la CADEG est cet instrument juridique de l'UA qui garantit le mieux l'alternance politique. Aux termes de son article 2, la CADEG promeut la tenue régulière d'élections transparentes, libres et justes dans le but non seulement de permettre aux Etats africains d'avoir des autorités et de gouvernements légitimes, mais aussi de permettre les changements démocratiques de gouvernement ${ }^{16}$. Ainsi tout change-

$11 \S 13$ de la Déclaration de l'UA sur la gouvernance démocratique...op. cit.

12 OUA, Mémorandum d'accord sur la stabilité, stabilité, développement et de la coopération en Afrique, Durban, 2002.

13 OUA, Déclaration sur le cadre pour une réaction de l'OUA face aux changements anticonstitutionnels de gouvernement, Lomé, le 12 juillet 2000.

$14 \S 8$ du préambule de la Charte africaine de la démocratie, des élections et de la gouvernance, Addis Abeba, 2007.

15 Art. 2 point 2 de la Charte africaine de la démocratie, des élections et de la gouvernance...op. cit.

16 Art. 2 point 3 de la Charte africaine de la démocratie, des élections et de la gouvernance...op. cit. 
ment démocratique inclut nécessairement l'alternance démocratique à terme. La CADEG prescrit que « l'accès au pouvoir et son exercice doivent se faire conformément à la constitution de l'Etat et au principe de l'Etat de droit ${ }^{17}$.

Trois autres dispositions de la CADEG garantissent l'alternance politique par la prescription et la proscription de certains comportements. Il s'agit des articles 5, 10 et 23. A l'article 5, la CADEG invite les Etats africains à prendre des mesures appropriées pour assurer le respect de l'ordre constitutionnel, plus particulièrement en ce qui concerne le transfert du pouvoir; en d'autres termes dans le cadre de l'alternance politique ${ }^{18}$. L'article 10 vise tout artifice juridique tendant à empêcher l'alternance politique. L'allusion est faite ici aux tripatouillages constitutionnels relatifs à la manipulation des dispositions constitutionnelles limitant le nombre et la durée des mandats présidentiels.

En effet, la CADEG oblige les Etats à renforcer le principe de la suprématie de la Constitution pour assurer que «...le processus d'amendement ou de révision de leur constitution repose sur un consensus national comportant, le cas échéant, le recours au référendum $^{19} \ldots$... Le consensus national pour la révision de la Constitution est un principe capital et est utilisé par les antirévisionnistes comme un argument de taille dans les débats politiques au sein des Etats africains confrontés aux velléités de modification ou de changement constitutionnels ${ }^{20}$.

En revanche, le recours au référendum est techniquement favorable aux partisans de la révision constitutionnelle lorsque l'on se situe dans le contexte politique de la RDC. Voici ce qu'écrit Evariste Boshab à ce sujet:

Le référendum pose [...] des difficultés quant à la capacité du peuple à se prononcer en connaissance de cause, notamment au vu des éléments qui lui sont fournis à cette fin. D'abord, fort souvent, la manière dont la question est élaborée, en matière de référendum, est entourée d'une certaine ambigü̈té. Elle ne permet pas à ceux appelés à se prononcer de saisir les vrais enjeux.

Tout se réduit, en définitive, à une sorte de plébiscite : on s'accorde plus sur la personnalité qui pose la question que sur la question elle-même. [...] Si la participation du citoyen au suffrage est sans conteste, le doute plane sur le caractère démocra-

17 Art. 3 point 2 de la Charte africaine de la démocratie, des élections et de la gouvernance...op. cit.

18 Art. 5 de la Charte africaine de la démocratie, des élections et de la gouvernance...op. cit.

19 Art. 10 points $1 \& 2$ de la Charte africaine de la démocratie, des élections et de la gouvernance... op. cit.

20 Il paraît un instrument à double tranchant. Le consensus peut être fabriqué et sans reposer sur l'assentiment réel du peuple souverain. Au Congo Brazzaville, profitant de la faiblesse de l'Opposition politique et de la Société civile, le Président Sassou Nguesso a changé de constitution pour se faire élire après un dialogue national qui n'a pas bénéficié d'un consensus national. Au Rwanda, le Président Paul Kagame, anticipant les événements et in tempore non suspecto (sic ?) a révisé la Constitution pour lui permettre d'être au pouvoir jusqu'en 2034. Dans ce pays où le régime est fortement autoritaire et intolérant, il est impossible d'obtenir le consensus national lorsqu'une bonne frange de la population n'a pas droit à la parole libre. 
tique, car, dès lors que se profilent plusieurs non-dits à travers des questions apparemment anodines, le plus grand perdant reste le peuple ${ }^{21}$.

Evariste Boshab a parfaitement raison lorsqu'il pense qu'un référendum organisé dans ce contexte ne sera pas démocratique à cause notamment des objectifs inavoués que poursuivent ses initiateurs. Ce qui inquiète le plus est que les électeurs sont souvent appelés à se prononcer sur des questions dont ils ne maitrisent pas les enjeux, le sens et la portée. Au bout du compte, les résultats du référendum avoisinent toujours les $100 \%$ sans qu'ils reflètent la réalité des urnes et ne soient conformes à la volonté des électeurs et du peuple tout entier.

L'article 23 de la CADEG indique des éléments constitutifs du changement anticonstitutionnel de gouvernement. Toute utilisation des moyens non démocratiques pour accéder ou se maintenir au pouvoir constitue un changement anticonstitutionnel de gouvernement. Deux de moyens énumérés dans la CADEG touchent à l'alternance politique. Il s'agit de « tout refus par un gouvernement en place de remettre le pouvoir au parti ou au candidat vainqueur à l'issue d'élections libres, justes et régulières ${ }^{22}$ » et de « tout amendement ou toute révision des Constitutions ou des instruments juridiques qui porte atteinte aux principes de l'alternance démocratique ${ }^{23} »$.

Dans le premier cas, le Gabon est concerné en dépit de l'arrêt de la Cour constitutionnelle dont on connaît l'ampleur de son inféodation aux régimes des Bongo (père et fils). Dans le deuxième cas, le Congo Brazzaville et le Rwanda sont concernés. Le changement de Constitution au Congo Brazzaville et l'amendement de la Constitution au Rwanda ont eu pour effet d'empêcher l'alternance démocratique. La RDC est sur ce schéma. Après avoir échoué de réviser la Constitution suite une vigilence accrue de la population, le régime de Joseph Kabila emploie l'artifice du dialogue politique national pour atteindre le même but.

En effet, le projet de l'accord politique proposé par la Facilitation du dialogue assurée par l'Union africaine porte des dispositions qui porte atteinte à l'ordre constitutionnel existant. Son chapitre IX relatif à la contuinité de l'Etat et à l'institution d'une période intérimaire prolonge le mandat du Président en fonction et du coup enfreint au principe de l'alternance qui devait intervenir le 19 décembre 2016. Ce projet d'accord constitue un renversement du régime constitutionnel en vigueur. Il prévoit entre autres la formation d'un gouvernement de large union nationale alors que la Constitution exige que le gouvernement soit formé par la Majorité parlementaire. Pendant que l'article 78 prescrit que le Premier ministre est choisi dans le rend de la Majorité parlementaire, le projet de l'accord politique prévoit le choix du Premier ministre au sein de l'Opposition. Il anéantit tous les pouvoirs de contrôle du Parlement sur le gouvernement et consacre une impunité généralisée sur une période sans limite connue.

21 Evariste Boshab, Entre la révision de la Constitution et l'inanition de la nation, Bruxelles, 2013, pp. 320-321.

22 Art. 23 point 4 de la Charte africaine de la démocratie, des élections et de la gouvernance...op. cit.

23 Art. 23 point 5 de la Charte africaine de la démocratie, des élections et de la gouvernance...op. cit. 
Dans ce ciel sombre pour l'alternance politique en Afrique, il rayonne des étoiles démocratiques comme le Bénin et le Sénégal qui en constitue le premier exemple sur le continent en 1980.

Tableau périodique de l'alternance politique au Sénégal depuis 1960

\begin{tabular}{|l|l|l|l|l|l|}
\hline $\begin{array}{l}\text { Noms du Pré- } \\
\text { sident de la Ré- } \\
\text { publique }\end{array}$ & $\begin{array}{l}\text { Date d'accès } \\
\text { au pouvoir }\end{array}$ & $\begin{array}{l}\text { Mode d'acces- } \\
\text { sion }\end{array}$ & $\begin{array}{l}\text { Date d'évic- } \\
\text { tion de la } \\
\text { fonction }\end{array}$ & $\begin{array}{l}\text { Mode d'évic- } \\
\text { tion }\end{array}$ & $\begin{array}{l}\text { Noms du Pré- } \\
\text { sident succes- } \\
\text { seur }\end{array}$ \\
\hline $\begin{array}{l}\text { Léopold Sedar } \\
\text { Senghor }\end{array}$ & $\begin{array}{l}5 \text { septembre } \\
1960\end{array}$ & $\begin{array}{l}\text { Election par un } \\
\text { collège électo- } \\
\text { ral }^{24}\end{array}$ & $\begin{array}{l}31 \text { décembre } \\
1980\end{array}$ & $\begin{array}{l}\text { Démission vo- } \\
\text { lontaire }\end{array}$ & Abdou Djouf \\
\hline Abdou Djouf & $\begin{array}{l}1 \mathrm{er} \text { janvier } \\
1980\end{array}$ & $\begin{array}{l}\text { Dauphin } \\
\text { constitutionnel } \\
\text { du Président en } \\
\text { exercice }\end{array}$ & 19 mars 2000 & $\begin{array}{l}\text { Défaite électo- } \\
\text { rale }\end{array}$ & Abdoulay Wade \\
\hline Abdoulay Wade & 19 mars 2000 & $\begin{array}{l}\text { Victoire électo- } \\
\text { rale }\end{array}$ & $\begin{array}{l}25 \text { mars } 2012 \\
\text { Victoire électo- } \\
\text { rale }\end{array}$ & $\begin{array}{l}\text { En cours } \\
\text { rale électo- }\end{array}$ & Macky Sall \\
\hline Macky Sall & 2 avril 2012 & ---------- & $----------~$ \\
\hline
\end{tabular}

Le Sénégal est le modèle par excellence de l'alternance politique en Afrique. A partir 1980 alors que plusieurs chefs d'Etat ayant conduit leurs peuples à l'indépendance s'accrochaient à leurs fauteuils présidentiels, Léopold Sedar Senghor avait volontairement quitté le pouvoir. Cet acte a cimenté la culture de l'alternance politique démocratique au Sénégal. Par deux fois, les Présidents en exercice ont perdu les élections et ont accepté la défaite.

$\mathrm{Au}$ niveau régional, les Communautés économiques régionales (CER) ont développé depuis la fin des années 1990 des activités politiques intenses dans les Etats membres. Elles ont été très actives dans le règlement des conflits armés et dans l'accompagnement des processus de transitions politiques dans leurs Etats membres, plus particulièrement en Afrique de l'Ouest et en Afrique australe ${ }^{25}$.

\section{B. L'alternance politique dans les chartes constitutives des Communautés économiques régionales (CER)}

La RDC est membre de trois CER que sont la Communauté économique des Etats de l'Afrique (CEEAC), le Common Market for Easthern and Southern Africa (COMESA) et la

24 Adrien Thouvenel-Avenas, L'alternance politique au Sénégal : 1980-2000, Master 2, Université Sorbonne Paris IV, 2007, http://www.memoireonline.com/11/07/691/alternance-politique-senegal-1980-2000.html (Consulté le 27 septembre 2016).

25 Voir dans ce sens, Pascal Sundi Mbambi P., La politique sécuritaire et de défense de la SADC et la crise congolaise, Congo-Afrique, n 396, (Juin-Juillet-Août 2005), pp. 357... et Dala Diana et Pascal Sundi Mbambi, Organisations régionales, conflits armés et missions de paix en Afrique : défis et perspectives, Congo-Afrique, n 407, (Septembre 2006), p. 280. 
Southern Africa Development Communauty $(\mathrm{SADC})^{26}$. De ces trois CER, seule la SADC a été active en matière politique dans les Etats membres. Dans le cadre cette étude, nous nous intéresserons à la SADC et à la contribution de la Conférence internationale sur la Région des Grands Lacs (CIRGL) à la promotion de l'alternance politique dans ses Etats membres.

\section{La SADC et l'alternance politique dans les Etats membres}

Le traité de la SADC prend en charge dans ses dispositions la question de la démocratie et de l'Etat de droit comme un des objectifs à atteindre au sein des Etats membres. Ces objectifs pourront être atteints par la promotion des valeurs politiques communes, des systèmes et d'autres valeurs partagées transmises à travers des institutions démocratiques, légitimes et efficaces et par la consolidation, la défense et le maintien de la démocratie, la paix, la sécurité et la stabilité ${ }^{27}$. Dans la même perspective, le protocole de la SADC en matière politique, de défense et de sécurité martèle sur le besoin et l'urgence pour développer les institutions et les pratiques démocratiques au sein des Etats membres de la SADC ${ }^{28}$. C'est dans cette optique que la SADC a édicté des principes régissant les élections démocratiques dans les Etats membres ${ }^{29}$.

Eu égard aux dispositions du Traité, du Protocole sur la coopération en matière politique, de défense et de sécurité et des principes régissant l'organisation des élections, on peut affirmer sans crainte d'être contredit que la SADC est une organisation internationale qui milite pour l'alternance politique dans ses Etats membres. C'est pour cette raison certainement que l'on retrouve un nombre élevé des anciens Présidents de la République dans cette région de l'Afrique ${ }^{30}$.

26 Balinngene Kahombo, La coopération judiciaire pénale fondée sur les traités de la CEEAC, du COMESA et de la SADC, Librairie africaine d'études juridiques, Volume 5, Août 2010, pp. 1-23; Anne-Marie Nsaka Kabunda, La problématique de l'affiliation multiple de la RDC aux organisations africaines : état de la question, conflits potentiels et solutions, Librairie africaine d'études juridiques, Volume 15, Août 2013, pp. 156...; Joseph Cihunda Hengelela, Intégration de la République Démocratique du Congo à la SADC : Traité, domaines de coopération et perspectives d'avenir, Librairie africaine d'études juridiques, Volume 5, Août 2010, p. 25.

27 Art. 4 et 5 point 1 a et b du Traité de la SADC, Windhoek, le 17 août 1992.

28 Protocole de coopération en matière politique, de défense et de sécurité, Blantyre, le 14 août 2001.

29 Claude Kirongozi Ichalanga, Le rôle de la SADC et de ses Etats membres dans le processus de démocratisation de la République Démocratique du Congo, in Grégoire Bakandeja wa Mpungu, André Mbata Betukumesu Mangu et Raoul Kienge-Kienge Intudi (dir.), Participation et responsabilité des acteurs dans un contexte d'émergence démocratique en République Démocratique du Congo. Actes des Journées scientifiques de la Faculté de Droit de l’Université de Kinshasa 18-19 juin 2007, Kinshasa, 2007, p. 240. Voir également, Joseph Cihunda Hengelela, Règlement des conflits au sein de la Communauté de développement de l'Afrique australe, Mémoire de DES, Kinshasa, 2012.

30 André Mbata B. Mangu, « Monarchies présidentielles et révisions constitutionnelles : le syndrome du troisième mandat ou d'une présidence à vie dans les Etats membres de l'Union africaine ", Revue africaine de la démocratie et de la gouvernance, Volume 1, n 1, 2014, p. 58 (pp.47-66); 
En ce qui concerne les élections, les Etats membres de la SADC ont adopté le protocole fixant les principes pour des élections démocratiques. Il s'agit d'un document contenant des « Principes pour la gestion, la surveillance et l'observation des élections dans la région de la SADC ». Ces principes visent à :

- assurer la pleine participation des citoyens dans le processus électoral et au-delà dans tout processus politique;

- assurer la pleine liberté d'association au sein des Etats membres;

- permettre l'organisation des élections dans un intervalle régulier conformément aux constitutions respectives des Etats membres;

- promouvoir la tolérance politique entre les acteurs politiques dans les Etats membres;

- offrir l'égale opportunité pour tous les partis politiques d'accéder aux médias;

- offrir l'égale opportunité pour l'exercice du droit de vote et du droit d'être élu;

- assurer l'indépendance et l'impartialité de l'institution chargée d'organiser les élections;

- contribuer à l'acceptation et au respect, par les partis politiques, de résultats des urnes proclamés par l'autorité électorale nationale compétente comme étant libre et juste en conformité avec la loi nationale.

Ces principes doivent s'analyser en termes d'obligations imposées aux Etats membres de la SADC et à la Communauté elle-même. L'obligation d'assurer la pleine participation des citoyens dans les processus politique en général et électoral en particulier est reprise dans de nombreux instruments juridiques comme un droit fondamental de tout citoyen ${ }^{31}$.

En effet, l'organisation politique de tout Etat est faite pour l'intérêt supérieur de tous les citoyens. Elle ne se conçoit pas contre une partie des citoyens ni en exclusion de ceux-ci. Les expériences de triste mémoire des périodes d'apartheid ou de ségrégation raciale ont démontré la vénalité de tels projets de société. Ainsi tout processus politique ou électoral doit s'assurer de la pleine participation de toutes les franges de la société concernée. Ce principe se recoupe avec celui visant à offrir une égale opportunité pour l'exercice du droit de vote et du droit d'être élu à tous les citoyens. C'est en favorisant une large participation

André Mbata B. Mangu, Hommages à Nelson Mandela. Leçons de leadership pour les dirigeants africains et du monde présents et à venir, Kinshasa, 2014, p. 24.

31 Art. 21 de la Déclaration universelle des droits de l'homme, Bulletin officiel, 1949, p. 1206 dispose que « Toute personne a le droit de prendre part à la direction des affaires publiques de son pays, soit directement, soit par l'intermédiaire de représentants librement choisis. Toute personne a droit à accéder, dans des conditions d'égalité, aux fonctions publiques de son pays ». Art. 25 du Pacte international relatif aux droits civils et politiques, New York, le 16 décembre 1966 dispose : « Tout citoyen a le droit et la possibilité, sans aucune des discriminations et sans restrictions déraisonnables : a) De prendre part à la direction des affaires publiques, soit directement, soit par l'intermédiaire de représentants librement choisis; b) De voter et d'être élu, au cours d'élections périodiques, honnêtes, au suffrage universel et égal et au scrutin secret, assurant l'expression libre de la volonté des électeurs; c) D'accéder, dans des conditions générales d'égalité, aux fonctions publiques de son pays ». Art. 13 de la Charte africaine des droits de l'homme et des peuples, Nairobi, le 1 juin 1981. Art. 3 et 4 de l'Acte constitutif de l'UA, Lomé, le 11 juillet 2000. Pour plus de détails, voir la Charte africaine de la démocratie, des élections et de la gouvernance, Addis-Abeba, 30 janvier 2007. 
qu'il est possible pour les citoyens d'exercer sans entrave leur droit de vote et de se porter candidat aux différents postes de responsabilités au sein de l'Etat.

L'obligation d'assurer la pleine liberté d'association au sein des Etats membres permet d'atteindre l'objectif relatif à la promotion des institutions et des pratiques démocratiques consacré dans le traité comme un des objectifs poursuivis par la SADC. Tout régime démocratique doit se caractériser entre autres par la liberté d'association reconnue aux citoyens. La liberté d'association favorise une bonne participation des citoyens à la vie publique. Ces derniers peuvent se constituer en groupements de la société civile pour influencer les décisions gouvernementales ou en partis politiques pour devenir des acteurs au pouvoir ou dans l'opposition politique. Lorsque la liberté d'association est pleinement assurée dans un Etat, elle débouche sur la tolérance politique entre les acteurs politiques et se manifeste notamment par le traitement équitable de tous les partis politiques surtout en ce qui concerne l'accès aux médias publics.

L'obligation d'organiser des élections dans un intervalle régulier suivant les dispositions constitutionnelles des Etats répond toujours à l'objectif de démocratisation des Etats membres de la SADC. Elle est une garantie à l'alternance politique au sein des Etats. L'exigence de la périodicité fait son occurrence à l'article 25 litera b du Pacte international relatif aux droits civils et politiques dispose :

Tout citoyen a le droit...De voter et d'être élu, au cours d'élections périodiques, honnêtes, au suffrage universel et égal et au scrutin secret, assurant l'expression libre de la volonté des électeurs.

Cette disposition est très explicite et vient au secours du principe de la SADC. Non seulement les élections sont organisées à des termes bien connus en conformité avec les dispositions de la Constitution et les lois en matière électorale mais aussi elles doivent être honnêtes et les résultats doivent refléter la vérité des urnes, c'est-à-dire la volonté du peuple telle qu'exprimée par le biais du bulletin de vote. C'est seulement si les élections sont transparentes que l'acceptation des résultats devient une obligation à respecter. Dans le cas contraire, une décision proclamant de faux résultats électoraux, quel que soit l'organe qui l'a prise, Commission électorale ou Cour constitutionnelle, perd tout fondement pour s'imposer aux parties constataires.

A propos de l'obligation d'assurer l'indépendance et l'impartialité de l'institution chargée d'organiser les élections, la SADC a pris en compte les demandes populaires visant à amputer certaines compétences électorales au Ministère de l'intérieur pour assurer la sincérité des opérations électorales. Cependant, les Organes de gestion des élections (OGE) ne sont pas à mesure de donner satisfaction aux attentes suscitées à l'occasion de leur création. C'est le constat que fait Pascal Kambale lorsqu'il note que

....tous les OGE sont confrontés à des problèmes similaires dans la gestion des élections. Parmi les plus importants, on peut citer la création et le maintien d'un registre électoral crédible, le coût élevé des élections, le manque de pouvoir pour sanctionner 
les fautes et le faible niveau d'implication des OGE dans la gestion des litiges électoraux ${ }^{32}$.

L'effort de mise en œuvre de ces principes dans les Etats membres a engendré la culture de l'alternance politique démocratique et pacifique dans la majorité des Etats membres de la SADC. Les pays suivants ont consolidé leur tradition de l'alternance politique et pacifique au pouvoir au niveau de la fonction présidentielle. Il s'agit : Du Botswana, de l'Ile Maurice, du Mozambique, de la Tanzanie, du Malawi, des Seychelles, de l'Afrique du Sud et de la Namibie.

Ce qui nous impose quelques illustrations à travers les tableaux suivants

Tableau périodique de l'alternance politique au Botswana

\begin{tabular}{|c|c|c|c|c|c|}
\hline $\begin{array}{l}\text { Noms du Pré- } \\
\text { sident de la Ré- } \\
\text { publique }\end{array}$ & $\begin{array}{l}\text { Date d'ac- } \\
\text { cession au } \\
\text { pouvoir }\end{array}$ & $\begin{array}{l}\text { Mode d'acces- } \\
\text { sion }\end{array}$ & $\begin{array}{l}\text { Date ces- } \\
\text { sation la } \\
\text { fonction }\end{array}$ & $\begin{array}{l}\text { Mode de ces- } \\
\text { sation de } \\
\text { fonction }\end{array}$ & $\begin{array}{l}\text { Noms du Pré- } \\
\text { sident succes- } \\
\text { seur }\end{array}$ \\
\hline $\begin{array}{l}\text { Sir Seretse Kha- } \\
\text { ma }\end{array}$ & $\begin{array}{l}30 \text { sep- } \\
\text { tembre } \\
1966\end{array}$ & Élection & $\begin{array}{l}13 \\
\text { juillet } 198 \\
0\end{array}$ & Décès & $\begin{array}{l}\text { Ketumile Ma- } \\
\text { sire }\end{array}$ \\
\hline Ketumile Masire & $\begin{array}{l}18 \text { juillet } \\
1980\end{array}$ & $\begin{array}{l}\text { Dauphin consti- } \\
\text { tutionnel \& } \\
\text { élection }\end{array}$ & $\begin{array}{l}1^{\text {er }} \text { avril } 19 \\
98\end{array}$ & $\begin{array}{l}\text { Démission vo- } \\
\text { lontaire }\end{array}$ & Festus Mogae \\
\hline $\begin{array}{l}\text { Festus } \\
\text { Mogae }\end{array}$ & $\begin{array}{l}1^{\text {er }} \text { avril } \\
1998\end{array}$ & $\begin{array}{l}\text { Dauphin consti- } \\
\text { tutionnel et élec- } \\
\text { tion }\end{array}$ & $\begin{array}{l}1^{\text {er }} \text { avril } 20 \\
08\end{array}$ & $\begin{array}{l}\text { Respect de la } \\
\text { limite des } \\
\text { mandats prési- } \\
\text { dentiels }\end{array}$ & $\begin{array}{l}\text { Seretse Khama } \\
\text { Ian Khama }\end{array}$ \\
\hline $\begin{array}{l}\text { Seretse Khama } \\
\text { Ian Khama }\end{array}$ & $\begin{array}{l}1^{\text {er }} \text { avril } \\
2008\end{array}$ & Election & En cours & & \\
\hline
\end{tabular}

32 Pascal Kambale, «Contribution des organes de gestion des élections à l'organisation d'élections crédibles en Afrique de l'Ouest ", in Ismailli Madior Fall, Hounkpe, Jinadu et Pascal Kambale, Organes de gestion des élections en Afrique de l'ouest. Une étude comparative de la contribution des commissions électorales au renforcement de la démocratie, Dakar, 2011, p. 9 (pp. 1-12). 
Tableau périodique de l'alternance politique en Namibie

\begin{tabular}{|l|l|l|l|l|l|}
\hline $\begin{array}{l}\text { Noms du Pré- } \\
\text { sident de la Ré- } \\
\text { publique }\end{array}$ & $\begin{array}{l}\text { Date de } \\
\text { prise de } \\
\text { fonction }\end{array}$ & $\begin{array}{l}\text { Mode d'acces- } \\
\text { sion }\end{array}$ & $\begin{array}{l}\text { Date de } \\
\text { cessation } \\
\text { de la } \\
\text { fonction }\end{array}$ & $\begin{array}{l}\text { Mode de ces- } \\
\text { sation }\end{array}$ & $\begin{array}{l}\text { Noms du Pré- } \\
\text { sident succes- } \\
\text { seur }\end{array}$ \\
\hline Sam Nujoma & $\begin{array}{l}21 \text { mars } \\
1990\end{array}$ & Election & $\begin{array}{l}21 \text { mars } \\
2005\end{array}$ & $\begin{array}{l}\text { Respect de la } \\
\text { limite des } \\
\text { mandats prési- } \\
\text { dentiels }\end{array}$ & $\begin{array}{l}\text { Hifikepunye } \\
\text { Pohamba }\end{array}$ \\
\hline $\begin{array}{l}\text { Hifikepunye Po- } \\
\text { hamba }\end{array}$ & $\begin{array}{l}21 \mathrm{mars} \\
2005\end{array}$ & Election & $\begin{array}{l}21 \\
\text { mars 2015 }\end{array}$ & Idem & Hage Geingob \\
\hline Hage Geingob & $\begin{array}{l}21 \text { mars } \\
2015\end{array}$ & Election & En cours & & \\
\hline
\end{tabular}

Tableau périodique de l'alternance politique au Malawi

\begin{tabular}{|c|c|c|c|c|c|}
\hline $\begin{array}{l}\text { Noms du Pré- } \\
\text { sident de la Ré- } \\
\text { publique }\end{array}$ & $\begin{array}{l}\text { Date de } \\
\text { prise de } \\
\text { fonction }\end{array}$ & $\begin{array}{l}\text { Mode d'acces- } \\
\text { sion }\end{array}$ & $\begin{array}{l}\text { Date de } \\
\text { cessation } \\
\text { de la fonc- } \\
\text { tion }\end{array}$ & $\begin{array}{l}\text { Mode de ces- } \\
\text { sation }\end{array}$ & $\begin{array}{l}\text { Noms du Pré- } \\
\text { sident succes- } \\
\text { seur }\end{array}$ \\
\hline $\begin{array}{l}\text { Hastings Kamu- } \\
\text { zu Banda }\end{array}$ & $\begin{array}{l}6 \text { juillet } \\
1966\end{array}$ & $\begin{array}{l}\text { Elections légis- } \\
\text { latives puis pré- } \\
\text { sidentielle }\end{array}$ & $\begin{array}{l}21 \\
\text { mai } 1994\end{array}$ & $\begin{array}{l}\text { Défaite élec- } \\
\text { torale }\end{array}$ & Bakili Muluzi \\
\hline Bakili Muluzi & $\begin{array}{l}21 \mathrm{mai} \\
1994\end{array}$ & Election & $\begin{array}{l}24 \\
\text { mai } 2004\end{array}$ & $\begin{array}{l}\text { Respect de la } \\
\text { limite des } \\
\text { mandats prési- } \\
\text { dentiels }\end{array}$ & $\begin{array}{l}\text { Bingu wa } \mathrm{Mu}- \\
\text { tharika }\end{array}$ \\
\hline $\begin{array}{l}\text { Bingu wa Mu- } \\
\text { tharika }\end{array}$ & $\begin{array}{l}24 \text { mai } \\
2004\end{array}$ & Election & $\begin{array}{l}6 \\
\text { avril } 2012\end{array}$ & Décès & Joyce Banda \\
\hline Joyce Banda & $\begin{array}{l}7 \text { avril } \\
2012\end{array}$ & $\begin{array}{l}\text { Dauphine } \\
\text { constitutionnelle }\end{array}$ & $\begin{array}{l}31 \text { mai } 201 \\
4\end{array}$ & $\begin{array}{l}\text { Défaite élec- } \\
\text { torale }\end{array}$ & $\begin{array}{l}\text { Peter Muthari- } \\
\text { ka }\end{array}$ \\
\hline Peter Mutharika & $\begin{array}{l}31 \text { mai } \\
2014\end{array}$ & Election & En cours & & \\
\hline
\end{tabular}


Tableau périodique de l'alternance politique en Afrique du Sud depuis 1994

\begin{tabular}{|l|l|l|l|l|l|}
\hline $\begin{array}{l}\text { Noms du Pré- } \\
\text { sident de la Ré- } \\
\text { publique }\end{array}$ & $\begin{array}{l}\text { Date de } \\
\text { prise de } \\
\text { fonction }\end{array}$ & $\begin{array}{l}\text { Mode d'acces- } \\
\text { sion }\end{array}$ & $\begin{array}{l}\text { Date de } \\
\text { cessation } \\
\text { de la fonc- } \\
\text { tion }\end{array}$ & $\begin{array}{l}\text { Mode de ces- } \\
\text { sation de la } \\
\text { fonction }\end{array}$ & $\begin{array}{l}\text { Noms du Pré- } \\
\text { sident succes- } \\
\text { seur }\end{array}$ \\
\hline $\begin{array}{l}\text { Frederick De } \\
\text { Clerk }\end{array}$ & $\begin{array}{l}15 \text { août } \\
1989\end{array}$ & $\begin{array}{l}\text { Victoire élec- } \\
\text { torale (sous } \\
\text { apartheid) }\end{array}$ & $\begin{array}{l}10 \text { mai } \\
1994\end{array}$ & $\begin{array}{l}\text { Défaite électo- } \\
\text { rale }\end{array}$ & $\begin{array}{l}\text { Nelson Man- } \\
\text { dela }\end{array}$ \\
\hline Nelson Mandela & 10 mai & $\begin{array}{l}\text { Victoire élec- } \\
\text { torale (à la fin } \\
\text { de l'apartheid) }\end{array}$ & $\begin{array}{l}14 \text { juin } \\
1999\end{array}$ & $\begin{array}{l}\text { Renonciation } \\
\text { au deuxième } \\
\text { mandat }\end{array}$ & Thabo Mbeki \\
\hline Thabo Mbeki & 1994 juin & $\begin{array}{l}\text { Victoire élec- } \\
\text { torale }\end{array}$ & $\begin{array}{l}25 \text { sep- } \\
\text { tembre } \\
2008\end{array}$ & $\begin{array}{l}\text { Démission vo- } \\
\text { lontaire suite } \\
\text { aux pressions } \\
\text { de l‘ANC }\end{array}$ & $\begin{array}{l}\text { Kgalema Mot- } \\
\text { lanthe }\end{array}$ \\
\hline $\begin{array}{l}\text { Kgalema Mot- } \\
\text { lanthe }\end{array}$ & $\begin{array}{l}25 \text { sep- } \\
\text { tembre }\end{array}$ & $\begin{array}{l}\text { Intirim en tant } \\
\text { que Vice-pré- } \\
\text { sident }\end{array}$ & $\begin{array}{l}\text { Election du } \\
\text { nouveau } \\
\text { Président } \\
\text { de la Répu- } \\
\text { blique }\end{array}$ & $\begin{array}{l}9 \text { mai } \\
2009\end{array}$ & Jacob Zuma \\
\hline Jacob Zuma & $\begin{array}{l}2008 \\
2009\end{array}$ & $\begin{array}{l}\text { Victoire élec- } \\
\text { torale }\end{array}$ & En cours & & \\
\hline
\end{tabular}

Hormis les deux monarchies de la sous-région qui ne sont pas concernées par l'alternance politique au niveau de la fonction présidentielle, les autres Etats membres comme le Zimbabwe, le Madagascar, l'Angola et la RDC peinent à vivre une alternance au pouvoir de manière démocratique et pacifique. La Zambie connaît une alternance politique depuis l'accession de Frederic Chiluba au pouvoir en 1991. Cette alternance perd de plus en plus son caractère pacifique avec les violences post-électorales. D'où la nécessité d'une recherche des conditions sociologiques pour une alternance politique démocratique.

\section{La CIRGL et l'alternance politique dans les Etats membres}

La CIRGL est la dernière Communauté économique régionale sur l'échiquier institutionnel panafricain. Elle est composée de 12 Etats depuis l'adhésion du Sud-Soudan. Née sur les cendres fumantes des atrocités incessantes sur les peuples de cette région d'Afrique. Elle a été conçue comme une solution durable aux conflits armés en Afrique des Grands Lacs. Ainsi la démocratie et la bonne gouvernance ont été identifiées comme faisant partie de la quête de l'établissement de la sécurité, de la stabilité et du développement.

Le Pacte sur la sécurité, la stabilité et le développement dans la région des Grands Lacs qui sert de cadre juridique à la Déclaration de Dar-es-Salaam, a entre autres pour objectifs de « créer des conditions de sécurité, de stabilité et de développement durables entre les 
Etats membres ${ }^{33} »$. Parmi ces conditions figurent la démocratie et la bonne gouvernance. Les Etats membres s'engageaient à respecter et à promouvoir la démocratie et la bonne gouvernance ${ }^{34}$, plus particulièrement à :

- respecter et à promouvoir les principes et les normes démocratiques;

- mettre en place des institutions de promotion de la bonne gouvernance, de l'Etat de droit et de respect des droits de l'homme à travers des systèmes constitutionnels fondés sur la séparation des pouvoirs, le pluralisme politique, l'organisation régulière d'élections démocratiques et crédibles, la gestion participative, transparente et responsable des affaires, des institutions et des biens publics.

Le protocole prévu pour appliquer ce pacte s'ouvre sur la consécration des principes de convergence constitutionnelle. Aux termes de l'article 12 de ce protocole, les Etats parties s'engagent à respecter les principes constitutionnels suivants :

- la séparation des pouvoirs exécutif, législatif et judiciaire;

- l'accession au pouvoir à travers des élections régulières, libres, honnêtes et transparentes;

- l'interdiction de tout changement anticonstitutionnel et de tout mode non démocratique d'accession ou de maintien au pouvoir;

- la participation populaire aux prises de décisions dans le strict respect des principes démocratiques;

- la décentralisation du pouvoir à tous les niveaux du gouvernement;

- l'apolitisme de l'armée;

- la laïcité de l'Etat et de ses institutions;

- le caractère national de l'Etat et de ses institutions;

- l'élimination de toute discrimination ethnique, religieuse, raciale, sexiste ou régionale;

- la parité entre les hommes et les femmes;

- le pluralisme politique;

- la liberté d'association, de réunion et de manifestation pacifique;

- la liberté de presse et d'expression; et

- la liberté de circulation impliquant en particulier l'interdiction de l'exil forcé.

Sans que l'alternance politique soit clairement mentionnée dans ce protocole, l'interprétation de ces principes permet d'affirmer que la CIRGL œuvre pour la promotion de l'alternance politique au sein des Etats membres. Cette alternance est garantie à deux niveaux. Dans les Etats où il est consacré le principe de la limitation constitutionnelle des mandats présidentiels, l'interdiction de tout changement constitutionnel et de tout mode non démocratique d'accession ou de maintien au pouvoir est une garantie juridique fondamentale à l'alternance politique. C'est le cas de la RDC, du Kenya, de la Tanzanie, de la Zambie, du Burundi et de la RCA.

33 Art. 2 (c) du Pacte sur la sécurité, la stabilité et le développement dans la région des Grands Lacs, Nairobi, le 15 décembre 2006.

34 Art. 6 du Pacte sur la sécurité,...op. cit. 
Il faut toute de suite signaler que ce principe a été violé en cette année 2016 par le Congo-Brazzaville et le Rwanda. Dans ces deux Etats membres de la CIRGL, des référendums populaires ont été organisés pour le changement de Constitution pour le Congo Brazzaville et pour réviser la Constitution pour le Rwanda. Dans les deux cas, les manipulations constitutionnelles ont permis au Président Sassou Nguesson de se maintenir au pouvoir et assure au Président Paul Kagame de rester au pouvoir au-delà de 2017.

Dans l'hypothèse des Etats où le nombre des mandats présidentiels n'est pas limité, l'alternance politique est garantie par le principe de l'accession au pouvoir à travers des élections régulières, libres, honnêtes et transparentes. Ceci ne peut constituer une garantie crédible à conditions que ces élections soient réellement libres et transparentes.

Le Protocole de la CIRGL sur la démocratie et la bonne gouvernance prescrit des dispositions juridiques pertinentes au sujet de l'organisation des élections. En effet, les Etats parties se sont engagés à garantir à tout citoyen remplissant les conditions requises le droit et la possibilité sans aucune discrimination de voter et d'être élu au cours d'élections périodiques, honnêtes au suffrage universel et égal et au scrutin secret assurant l'expression de la volonté des électeurs ${ }^{35}$.

Le Protocole interdit de réforme électorale substantielle dans les six mois précédant les élections sans le consentement d'une large majorité des autres acteurs politiques ${ }^{36}$. Les élections à tous les niveaux doivent avoir lieu aux dates ou périodes fixées par la Constitution ou les lois électorales ${ }^{37}$. Les Etats parties se sont engagés à prendre des mesures appropriées pour garantir aux femmes, à égalité avec les hommes, le droit de voter et d'être élus lors des élections, de participer à la formulation et à la mise en œuvre des politiques gouvernementales et de remplir de fonctions publiques à tous les niveaux de prise de déci$\operatorname{sion}^{38}$.

L'indépendance des organes électoraux doit être assurée par les Etats parties à travers une concertation nationale appropriée pour déterminer la nature et la forme desdits organes. Ces organes doivent, dans leur composition, refléter le principe de parité entre les hommes et les femmes ${ }^{39}$.

Les Etats parties doivent s'assurer de l'établissement d'un système d'état civil fiable et stable. Ils doivent s'engager à coopérer dans ce domaine par des échanges d'expériences et au besoin par une assistance technique ${ }^{40}$. Les organes électoraux établiront les listes électorales de manière transparente et crédible. Les parties prenantes peuvent les consulter au tant

37 Art. 5 du Pacte sur la sécurité,...op. cit.

38 Art. 6 du Pacte sur la sécurité,...op. cit.

39 Art. 7 du Pacte sur la sécurité,...op. cit.

40 Art. 8 du Pacte sur la sécurité,...op. cit. 
que de besoin. Ce qui garantit le principe de la transparence ${ }^{41}$. Malgré ces dispositions juridiques, le seul exemple crédible de l'alternance démocratique demeure la Tanzanie.

\section{Tableau périodique de l'alternance politique en Tanzanie}

\begin{tabular}{|c|c|c|c|c|c|}
\hline $\begin{array}{l}\text { Noms du Pré- } \\
\text { sident de la } \\
\text { République }\end{array}$ & $\begin{array}{l}\text { Date de prise de } \\
\text { fonction }\end{array}$ & $\begin{array}{l}\text { Mode d'ac- } \\
\text { cession }\end{array}$ & $\begin{array}{l}\text { Date de cessation } \\
\text { de la fonction }\end{array}$ & $\begin{array}{l}\text { Mode de } \\
\text { cessation } \\
\text { de la fonc- } \\
\text { tion }\end{array}$ & $\begin{array}{l}\text { Noms du } \\
\text { Président } \\
\text { successeur }\end{array}$ \\
\hline $\begin{array}{l}\text { Julius } \\
\text { Nyerere }\end{array}$ & 29 octobre 1964 & Election & 5 novembre 1985 & $\begin{array}{l}\text { Retraite po- } \\
\text { litique }\end{array}$ & $\begin{array}{l}\text { Ali Hassan } \\
\text { Mwinyi }\end{array}$ \\
\hline $\begin{array}{l}\text { Ali Hassan } \\
\text { Mwinyi }\end{array}$ & 5 novembre 1985 & $\begin{array}{l}\text { Succession } \\
\text { en tant que } \\
\text { vice-pré- } \\
\text { sident puis } \\
\text { élection }\end{array}$ & 23 novembre 1995 & & $\begin{array}{l}\text { Benjamin } \\
\text { Mkapa }\end{array}$ \\
\hline $\begin{array}{l}\text { Benjamin } \\
\text { Mkapa }\end{array}$ & 23 novembre 1995 & Election & 21 décembre 2005 & $\begin{array}{l}\text { Respect de } \\
\text { la limite des } \\
\text { mandats } \\
\text { présiden- } \\
\text { tiels }\end{array}$ & $\begin{array}{l}\text { Jakaya } \\
\text { Kikwete }\end{array}$ \\
\hline $\begin{array}{l}\text { Jakaya } \\
\text { Kikwete }\end{array}$ & 21 décembre 2005 & Election & 5 novembre 2015 & Idem & $\begin{array}{l}\text { John } \\
\text { Magufuli }\end{array}$ \\
\hline $\begin{array}{l}\text { John } \\
\text { Magufuli }\end{array}$ & 5 novembre 2015 & Election & en & & \\
\hline
\end{tabular}

\section{Conclusion}

Il a été démontré tant en théorie qu'en pratique que l'alternance politique au sein des Etats est un gage de la paix, de la stabilité nécessaire au développement économique, social et culturel. L'expérience africaine nous enseigne également que le maintien au pouvoir d'un seul homme est une source des conflits et de régression économique, sociale et culturelle. Il est clair aussi que l'obstination à vouloir par tous les moyens à se cramponner au pouvoir finit par plonger le pays dans le chaos.

Pour éviter aux pays et aux peuples d'Afrique les scènes macabres d'apocalypse et leurs permettre de gagner le temps perdu sur la trajectoire du développement, les Président de la République devaient humblement et patriotiquement s'engager à quitter le pouvoir à l'issue d'une défaite électorale ou au terme de leur dernier et ultime mandat présidentiel. Que les Etats où le principe de limitation des mandats à la fonction présidentielle n'existe pas ou plus, qu'il soit instauré ou réinstauré. Il s'agit de consolider les principes démocratiques et de l'Etat de droit sans lesquels il n'y aura ni développement ni émergence à l'horizon 2030 .

41 Art. 9-10 du Pacte sur la sécurité,...op. cit. 
Compte tenu de la situation interne de chaque pays, les organisations internationales africaines ont un rôle à jouer pour favoriser l'alternance politique au sein des Etats membres. Elles doivent respecter leurs textes juridiques en la matière et mener une action en deux directions. D'une part, elles peuvent s'appuyer sur la dynamique interne pour faire pression sur les Présidents de la République en vue de respecter le principe de l'alternance au pouvoir. Cela appelle à une rupture de la solidarité négative qui fait de la Conférence de l'UA un syndicat des chefs d'Etat comme à l'époque de l'OUA. D'autre part et en aval, elles peuvent se servir des mécanismes du CPS pour considérer toute atteinte à l'alternance politique comme une menace à la paix et à la sécurité internationales. Ce qui devrait justifier le développement des OMP avec des mandats électoraux plus robustes et clairs.

La surveillance du régime démocratique instituée en Afrique n'est pas une violation du principe de la souveraineté des Etats. Au contraire, elle est son affirmation absolue et un mécanisme pour sa matérialisation. Dans un processus assez avancé du délitement de l'Etat, la souveraineté du peuple a été confisquée par un individu (ou un groupe d'individus), Président de la République, au détriment du peuple. Titulaire incontesté de la souveraineté, le peuple est souvent réprimé lorsqu'il revendique l'exercice de sa souveraineté. Au nom de l'obligation de protéger, les organisations internationales devaient intervenir pour respecter le droit des peuples à l'alternance politique au sein de leurs Etats membres.

Les dispositions du droit international promouvant l'alternance politique dans les Etats ont actuellement un écho favorable dans beaucoup de pays africains où les citoyens veulent en " finir avec les démocraties de façade ${ }^{42}$ et mettre un terme à la présidence monarchique ou à vie. En Afrique, avec la révolution populaire ayant renversé le régime de l'ancien Président Blaise Compaoré, l'appel à la limitation des mandats présidentiels a été lancé par l'opinion publique à l'UA. Celui-ci sera entendu lorsqu'il sera inséré dans la CADEG comme une disposition obligeant les Etats membres à limiter le nombre des mandats présidentiels dans leurs chartes constitutionnelles respectives.

La RDC a ratifié et/ou adhéré aux instruments juridiques de l'UA et de la SADC relatifs à l'alternance politique. Au sujet de la CADEG, la RDC l'a signé mais ne l'a pas encore ratifiée. En tant que Etat signataire de la Charte, le droit international lui impose de se s'abstenir de poser des actes qui vident ce traité de sa substance, même avant qu'il ne l'ait ratifié. Au cas contraire, elle aidera à confirmer les tentatives de retournements autoritaires qui ont commencé à élire domicile dans le chef des dirigeants politiques actuels. 


\section{Annexe}

Tableau périodique de l'alternance politique en RDC depuis 1960 (non démocratique)

\begin{tabular}{|c|c|c|c|c|c|c|}
\hline & $\begin{array}{l}\text { Noms du Pré- } \\
\text { sident de la Ré- } \\
\text { publique }\end{array}$ & $\begin{array}{l}\text { Date de prise } \\
\text { de fonction }\end{array}$ & $\begin{array}{l}\text { Mode d'ac- } \\
\text { cession }\end{array}$ & $\begin{array}{l}\text { Date d'évic- } \\
\text { tion de la } \\
\text { fonction }\end{array}$ & $\begin{array}{l}\text { Mode } \\
\text { d'éviction }\end{array}$ & $\begin{array}{l}\text { Noms du Pré- } \\
\text { sident succes- } \\
\text { seur }\end{array}$ \\
\hline 1 & $\begin{array}{l}\text { Joseph Kasavu- } \\
\text { bu }\end{array}$ & 1960 & $\begin{array}{l}\text { Election par } \\
\text { le Congrès } \\
\text { parlemen- } \\
\text { taire }\end{array}$ & $\begin{array}{l}24 \text { novembre } \\
1965\end{array}$ & $\begin{array}{l}\text { Coup d'Etat } \\
\text { militaire }\end{array}$ & $\begin{array}{l}\text { Joseph Désiré } \\
\text { Mobutu }\end{array}$ \\
\hline 2 & $\begin{array}{l}\text { Joseph Désiré } \\
\text { Mobutu }\end{array}$ & $\begin{array}{l}24 \text { novembre } \\
1965\end{array}$ & $\begin{array}{l}\text { Coup d'Etat } \\
\text { militaire }\end{array}$ & 17 mai 1997 & $\begin{array}{l}\text { Coup d'Etat } \\
\text { militaire }\end{array}$ & $\begin{array}{l}\text { Laurent-Désiré } \\
\text { Kabila }\end{array}$ \\
\hline 3 & $\begin{array}{l}\text { Laurent-Désiré } \\
\text { Kabila }\end{array}$ & $\begin{array}{l}\text { Laurent-Dési- } \\
\text { ré Kabila }\end{array}$ & $\begin{array}{l}\text { Coup d'Etat } \\
\text { militaire }\end{array}$ & $\begin{array}{l}16 \text { janvier } \\
2001\end{array}$ & Assassinat & $\begin{array}{l}\text { Joseph Kabila } \\
\text { Kabange }\end{array}$ \\
\hline 4 & $\begin{array}{l}\text { Joseph Kabila } \\
\text { Kabange }\end{array}$ & $\begin{array}{l}26 \text { janvier } \\
2001\end{array}$ & $\begin{array}{l}\text { Désignation } \\
\text { en tant que } \\
\text { fils de } \\
\text { Laurent Dé- } \\
\text { siré Kabila, } \\
\text { puis élection } \\
\text { en } 2006 \text { et } \\
\text { réélection en } \\
2011 .\end{array}$ & $\begin{array}{l}\text { Fonction en } \\
\text { cours }\end{array}$ & $\begin{array}{l}\text { L'échéance } \\
\text { de son } \\
\text { deuxième et } \\
\text { dernier man- } \\
\text { dat le } 19 \text { dé- } \\
\text { cembre } 2016\end{array}$ & 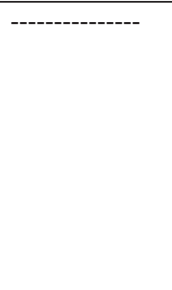 \\
\hline
\end{tabular}

Il y a lieu de signaler que la RDC n'a jamais vécu une expérience d'alternance politique démocratique et pacifique. Le transfert de pouvoir s'est réalisé jusqu'à présent par des moyens non constitutionnels et violents. Le tout dernier transfert des pouvoirs présidentiels s'était réalisé à la suite de l'assassinat du Président en fonction le 16 janvier 2001. 